\title{
Normal Continuous Cardiotocography Pattern
}

National Cancer Institute

\section{Source}

National Cancer Institute. Normal Continuous Cardiotocography Pattern. NCI Thesaurus.

Code C111771.

Normal fetal heart pattern. Strongly predictive of normal acid-base status at time of observation. Characterized by baseline fetal heart rate 110 to 160 beats per minute, moderate baseline variability, absent late or variable decelerations, absent or present early decelerations, and absent or present accelerations. 